\title{
Optimization of Visual Evoked Potential (VEP) Recording Systems
}

\author{
Rustum Karanjia, Donald G. Brunet, Martin W. ten Hove
}

\begin{abstract}
Objective: To explore the influence of environmental conditions on pattern visual evoked potential (VEP) recordings. Methods: Fourteen subjects with no known ocular pathology were recruited for the study. In an attempt to optimize the recording conditions, VEP recordings were performed in both the seated and recumbent positions. Comparisons were made between recordings using either LCD or CRT displays and recordings obtained in silence or with quiet background music. Paired recordings (in which only one variable was changed) were analyzed for changes in P100 latency, RMS noise, and variability. Results: Baseline RMS noise demonstrated a significant decrease in the variability during the first $50 \mathrm{msec}$ accompanied by a $73 \%$ decrease in recording time for recumbent position when compared to the seated position $(\mathrm{p}<0.05)$. Visual evoked potentials recorded using LCD monitors demonstrated a significant increase in the P100 latency when compared to CRT recordings in the same subjects. The addition of background music did not affect the amount of RMS noise during the first $50 \mathrm{msec}$ of the recordings. Conclusion: This study demonstrates that the use of the recumbent position increases patient comfort and improves the signal to noise ratio. In contrast, the addition of background music to relax the patient did not improve the recording signal. Furthermore, the study illustrates the importance of avoiding low-contrast visual stimulation patterns obtained with LCD as they lead to higher latencies resulting in false positive recordings. These findings are important when establishing or modifying a pattern VEP recording protocol.
\end{abstract}

RÉSUMÉ: Optimisation des systèmes d'enregistrement des potentiels évoqués visuels. Objectif : Le but de notre étude était d'explorer l'influence des conditions environnementales sur le tracé d'enregistrement de potentiels évoqués visuels (PEV). Méthodes : Quatorze sujets sans pathologie oculaire connue ont été recrutés. Afin d'optimiser les conditions d'enregistrement, les enregistrements des PEV ont été effectués en position assise et en position couchée. Nous avons comparé les enregistrements faits soit avec un afficheur LCD ou un afficheur CRT et les enregistrements obtenus en silence ou avec une musique de fond douce. Nous avons évalué les changements de la latence P100, du bruit rms et de la variabilité dans des enregistrements appariés (dont seulement une variable était modifiée). Résultats : Nous avons observé une diminution significative de la variabilité du bruit rms de base pendant les premières 50 msec accompagnée d'une diminution de $73 \%$ du temps d'enregistrement en position couchée par rapport à la position assise $(\mathrm{p}<0,05)$. Nous avons observé une augmentation significative de la latence P100 des PEV enregistrés au moyen d'afficheurs LCD par rapport aux enregistrements CRT chez les mêmes sujets. De plus, la musique de fond n'a pas modifié la quantité de bruit rms pendant les premières $50 \mathrm{msec}$ d'enregistrement. Conclusion : Cette étude démontre que l'utilisation de la position couchée augmente le confort des patients et améliore le ratio du signal par rapport au bruit. Par contre, l'ajout de musique de fond pour détendre le patient n'a pas amélioré le signal. De plus, l'étude démontre l'importance d'éviter les modes de stimulation visuelle à faible contraste obtenus avec le LCD parce qu'ils entraînent des latences plus élevées, ce qui donne des enregistrements faussement positifs. Ces constatations sont importantes pour établir ou modifier un protocole d'enregistrement de PEV.

Can. J. Neurol. Sci. 2009; 36: 89-92

Visual evoked potentials (VEP) are electrophysiological cortical activity evoked by visual stimuli and are commonly used as an objective, non-invasive method for analyzing visual information processing. There are several different types of VEP recordings, however, for most clinical purposes the pattern reversal VEP is the preferred modality because of its consistent waveform and latency. ${ }^{1,2}$ The evoked potential response is dependent on several factors including: the mean and background luminance, check size, reversal rate, pattern contrast, refractive error, quality of fixation and pupil size..$^{2,3}$

Published standards for VEP recording technique are available from the International Society for Clinical Electrophysiology of Vision (ISCEV) that includes information for the calibration of visual display monitors ${ }^{4}$ and standards for pattern reversal stimuli. ${ }^{2}$ Some parameters that affect pattern reversal VEP recordings are programmable such as check size and reversal rate, while others such as mean luminance are equipment dependent and must be calibrated in order to reduce

\footnotetext{
From the Departments of Ophthalmology (RK, MtH) and Division of Neurology, Department of Medicine (DB), Queen's University, Kingston, Ontario, Canada. Received January 31, 2008. Final Revisions Submitted August 6, 2008. Correspondence to: Martin ten Hove, Departments of Ophthalmology and Division of Neurology, Department of Medicine, Queen's University, Kingston, 166 Brock Street, Kingston, Ontario, K7L 5G2, Canada.
} 
the variability in latency. ${ }^{4}$ The guidelines recommend a mean luminance of at least $100 \mathrm{~cd} / \mathrm{m}^{2}$ and VEP systems are developed to meet these requirements. While commercially available VEP systems employ conventional cathode-ray tube (CRT) monitors, advances in liquid crystal display (LCD) technology have resulted in commercially available LCD displays which fulfill the ISCEV requirements (i.e. mean luminance $>100 \mathrm{~cd} / \mathrm{m}^{2}$ and contrast ratio of 1:1500). Some lab operators may thus be tempted to replace old CRT monitors with new LCD monitors.

Investigators agree that patient preparation is an important part of the clinical protocol. ${ }^{2}$ The ISCEV recommends that "care must be taken to have the patient in a comfortable, well supported position to minimize muscle and other artifacts", 2 however, no specific recommendations are provided as to how this should be accomplished.

In this study we sought to address two aspects of the ISCEV guidelines. First we compared the effect of switching the stimulus monitor from a conventional CRT to a LCD by measuring the effect on P100 latency. Secondly, we examined the effects of recumbent patient positioning and background music on the quality and variability of the pattern VEP signal.

\section{Methods}

Fourteen subjects, seven males and seven females, between 22 and 47 years-of-age with no known ocular pathology were recruited for the study. All subjects had a best corrected visual acuity of 6/6. Recordings were preformed under photopic conditions using the UTAS visual diagnostic system (LKC Technologies). Subjects were placed in an ophthalmologic examining chair one meter away from the stimulus screen. Recordings were made with ambient lighting $\left(50 \mathrm{~cd} / \mathrm{m}^{2}\right)$ and subjects were provided with 15 minutes to acclimatize to the lighting level, during which time gold disc scalp electrodes were placed at $\mathrm{Oz}$ (active), Fz (reference), and A1 or A2 (ground).
Three different check sizes ( $16 \times 16,64$ x 64, and $128 \times 128)$ were utilized. An average of 80 sweep tracings for each condition was analyzed. Pairs of recordings (in which only one variable was changed) were analyzed for changes in the P100 latency, background RMS noise levels (paired t-test), and signal variability (F-test) during the first 50 milliseconds. Each recording session was limited to one hour to ensure subjects were not fatigued. As a result not all experiments were conducted on all subjects. In addition, the sequence of the experiments was varied to negate any potential sequence bias. Recordings from two different sessions with the same subject were used to ensure that the results were reproducible. Subjects were observed by the examiner for movement and recordings were analyzed for a single, mono-phasic, $\mathrm{P} 100$ peak during the recording sessions by the LKC software. Recordings which did not meet these requirements were discarded and repeated.

Sixty pairs of recordings from subjects in the recumbent or seated position were compared. The screen to subject distance was held constant for the seated and recumbent position for each subject. Subjects were placed in the recumbent position with their heads at a 30 degree incline. Care was taken to ensure that the adjustable headrest comfortably supported the subjects' head and neck.

The effect of music was assessed in 30 pairs of recordings obtained in silence or with background classical music. The music was played through a computer using 15-watt desktop speakers. The volume was maintained at a quite background level in all recording pairs.

Thirty-two pairs of recordings using either LCD or CRT displays were also assessed. Three different CRT monitors were used: an AOC 7Glr, a Sony CPD-210GS and the LKC supplied stimulus monitor. Two different 17-inch LCD monitors were used, a Samsung $170 \mathrm{~N}$ and a LG L1752TX with mean luminances of $125 \mathrm{~cd} / \mathrm{m}^{2}$ and $150 \mathrm{~cd} / \mathrm{m}^{2}$ and contrast ratios of

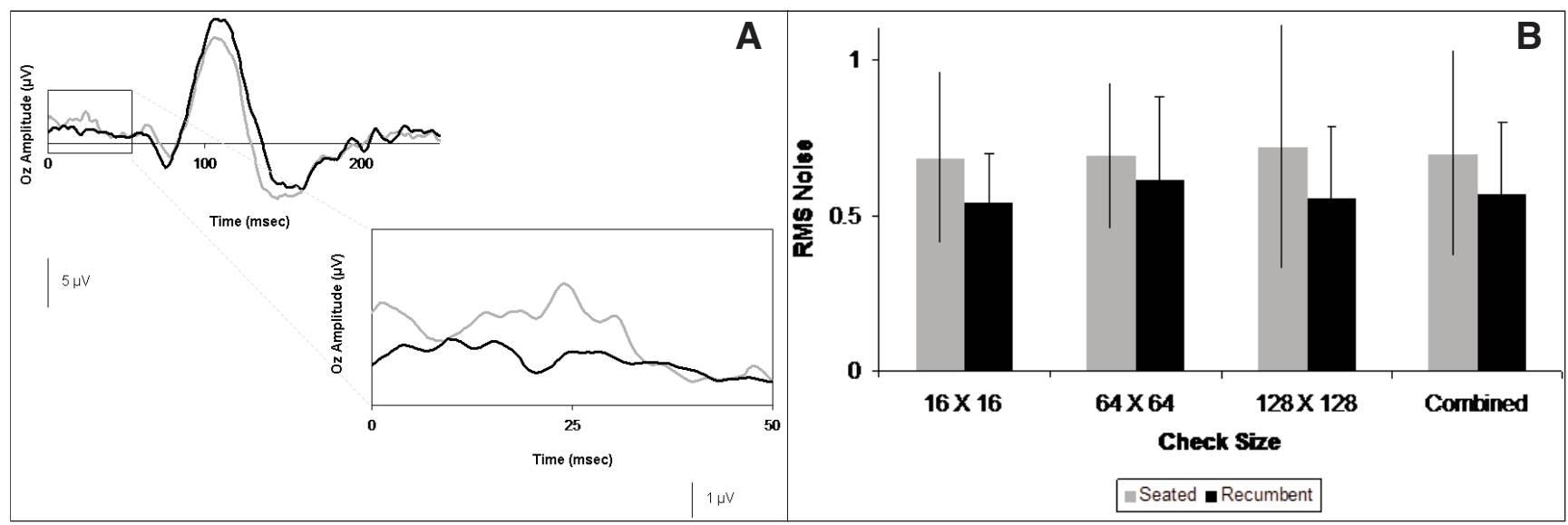

Figure 1: Two recordings in the seated and reclined positions in the same subject with magnified responses from the first 50 msec. A: There was a significant decrease in the amount of variability in the recumbent position when compared to the seated position in $57 \%$ of paired responses versus only $10 \%$ of paired responses favoring the seated position $(F$-test $<0.05)$. There was no significant difference in one third of responses. B: Assessment of the average RMS noise in the recumbent and seated positions demonstrated a significant decrease in the variability during the first 50 msec for all three check sizes and the average of all three check sizes (combined). (FTE) discounts productivity variables, notably sex and age. 


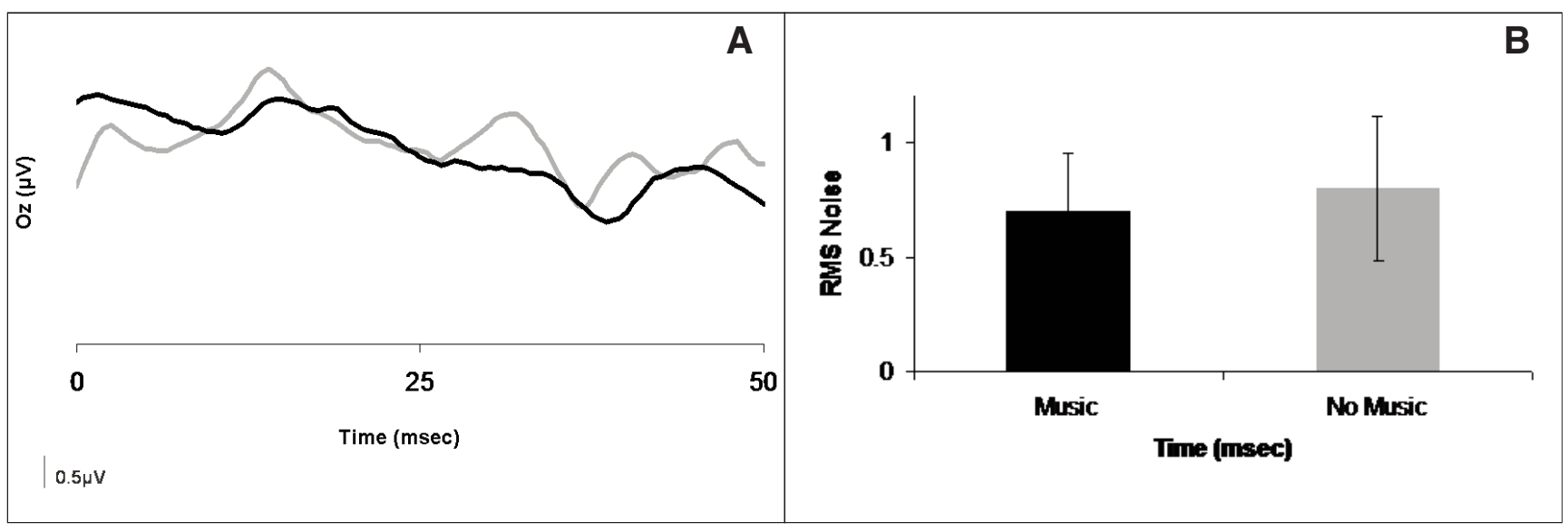

Figure 2: A: The addition of background classical music did not change variability during the first 50 msec (F-test $>0.05)$. B: There was also no change in the amount of RMS noise in the presence of background music $(p>0.3, n=30)$.

450:1 and 1400:1 respectively. The stimulus was output to both the CRT and LCD monitor simultaneously from the computer at identical refresh rates using a one to two VGA splitter with built in amplifier. The power cable to the monitor which was not being used was switched off to ensure background lumination was equal in both sets of experiments. Monitors were manually repositioned to ensure that they were centered appropriately one meter away and orthogonal to the patient's line of sight.

\section{RESULTS}

\section{Recumbent versus seated position}

Analysis of the first $50 \mathrm{msec}$ of the responses demonstrated a significant decrease in the amount of variability in the recumbent position when compared to the seated position in $57 \%$ of paired responses versus $10 \%$ of paired responses favoring the seated position (F-test <0.05) (Figure 1A). There was no significant difference in one third of responses. Assessment of the average RMS noise in 60 pairs of tracings in the recumbent and seated positions demonstrated a significant decrease in the variability during the first $50 \mathrm{msec}$ for all three check sizes (Figure 1B; * $\mathrm{p}<0.05)$.

\section{Music and $V E P$ recordings}

The addition of background classical music did not significantly alter the amount of RMS noise in the seated or recumbent positions ( $\mathrm{p}>0.3, \mathrm{n}=30)$. There was also no change in the variability during the first $50 \mathrm{msec}$ in all recording pairs (Ftest $>0.05$ ) (Figure 2A). There was no significant difference in the average RMS noise in sixty pairs of recordings in both the seated and recumbent positions in the presence or absence of background music $(\mathrm{p}>0.3)$ (Figure $2 \mathrm{~B})$.

\section{LCD versus CRT monitors}

The P100 latency was measured by the proprietary LKC software and a custom algorithm which detected the point of highest inflection point. There was a significant delay in the P100 (latency increase of $>25 \mathrm{msec}$ ) with both LCD monitors when compared to CRT recordings in the same subjects $(\mathrm{p}<0.05$, $\mathrm{n}=32$ ) (Figure 3). Similarly, the use of a low contrast CRT monitor also delayed the P100 response (data not shown).

\section{Discussion}

The ISCEV suggests care should be taken to decrease amount of noise arising from $60 \mathrm{~Hz}$ power supplies, occipital muscle tension and extraneous cortical activity. However, there is no standardized method to accomplish this. ${ }^{2}$ Our study demonstrates that placing the subject in the recumbent position does decrease the amount of noise from extraneous sources. Decreasing RMS noise allows for more accurate calculations of P100 amplitude. The recumbent position also decreased the number of repeated recordings required to obtain a good quality recording from 41 to 11 , (resulting in a $73 \%$ reduction in recording time). The addition of background music did not have any measurable effect on extraneous noise.

Our study also demonstrated the importance of the monitor type and contrast level affects the reliability of the VEP recordings. Low-contrast stimulation with either an LCD or older CRT monitor leads to prolonged latencies - potentially resulting in false positive recordings. While this is not surprising given that the older CRT monitors have low mean luminance levels (between $25 \mathrm{~cd} / \mathrm{m}^{2}$ and $50 \mathrm{~cd} / \mathrm{m}^{2}$ ), it also occurred with LCD monitors despite their mean luminances of greater than $100 \mathrm{~cd} / \mathrm{m}^{2}$.

The refresh rate of the screen is another variable that can impact the latency of the recording. Although the LCD monitors have a faster refresh rate $(8 \mathrm{~ms}=125 \mathrm{~Hz})$, the refresh rates in this experiment were intentionally controlled with a video splitter (60 $\mathrm{Hz}$ ) feeding both the LCD and CRT monitors. Increasing the video card output to $125 \mathrm{~Hz}$ was not permitted by the operating system used in our set up, nor by the video drivers for both monitors tested.

The contrast ratio of the monitors is another important consideration. The theoretical maximum contrast ratio which could be obtained from the LKC CRT monitor was 1:20,000. This is significantly higher than the reported contrast ratio of the 


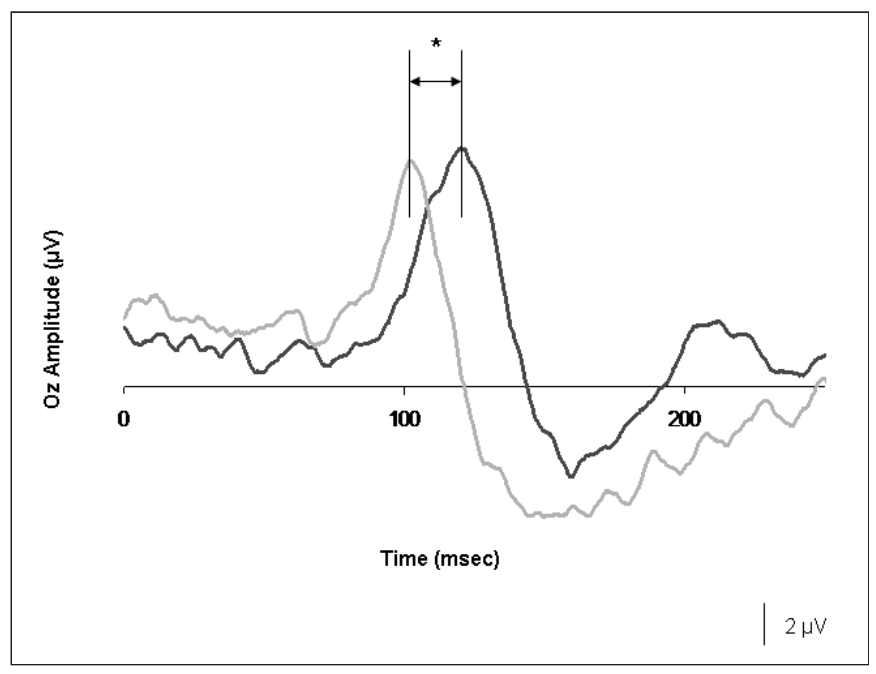

Figure 3: Paired recordings using LCD/CRT monitors. There was a significant increase in the P100 latency (increase of $>25 \mathrm{msec}$ ) with the $L C D$ monitor ( $L G$ L1752TX) when compared to CRT recordings in the same subjects $(* p<0.05, n=32)$. Typical pair of recordings from one subject. shown the simple substitution of an LCD monitor for a CRT monitor has the potential to cause prolonged latencies, suggesting that recalibration of the recordings systems would be necessary before this substitution could be made.

The changes in latency observed in this study are consistent with calibration issues identified by ISCEV. Sources of extraneous noise in VEP recordings such as cervical musculature can also be reduced through alterations in patient positioning. This study demonstrates that incorporation of the recumbent patient position into recording protocols can improve both recording quality and efficiency. Finally, it is imperative that new equipment display monitors be thoroughly tested before being integrated into a VEP recording protocol.

\section{REFERENCE}

1. Guideline 9B: Guidelines on visual evoked potentials. J Clin Neurophysiol. 2006 Apr;23(2):138-56.

2. Odom JV, Bach M, Barber C, Brigell M, Marmor MF, Tormene AP, et al. Visual evoked potentials standard (2004). Doc Ophthalmol. 2004 Mar;108(2):115-23.

3. Weinstein GW, Odom JV, Cavender S. Visually evoked potentials and electroretinography in neurologic evaluation. Neurol Clin. $1991 \mathrm{Feb} ; 9(1): 225-42$.

4. Brigell $\mathrm{M}$, Bach $\mathrm{M}$, Barber $\mathrm{C}$, Kawasaki K, Kooijman A. Guidelines for calibration of stimulus and recording parameters used in clinical electrophysiology of vision. Calibration Standard Committee of the International Society for Clinical Electrophysiology of Vision (ISCEV). Doc Ophthalmol. 1998; 95(1):1-14.
LCD monitor tested which was only 1:1400. Furthermore, the actual value was probably lower due to measured contrast ratio is reported for full-white and full-black screens. The use of a checkerboard pattern lowers the contrast ratio because light bleeds from white boxes to dark boxes. In addition, unlike CRT monitors, LCD monitors do not switch between colors with uniform speed. As a result the effective refresh rate from pure black to pure white of the LCD monitor is less than that of the CRT monitor. This may result in a flash artifact that could also contribute to the prolongation of the P100 latency. For all of these reasons, LCD monitors currently cannot be substituted for CRT monitors when performing VEP studies. However, given the rapid rate of advancement in computer technology, LCD design may soon overcome these technical shortcomings and become a reliable way to produce VEP stimuli.

The purpose of this study was to look for potential improvements in VEP recording quality by using the recumbent position or background music. The combination of background music and the recumbent position was not studied as there was no significant improvement found with background music alone. The significant decrease in recording time and decrease in RMS noise associated with the recumbent position provides good incentive for the incorporating this position into VEP recording protocol. Ideally, the stimulus display monitor could be suspended with a moveable ceiling mount to facilitate this adaptation. Unfortunately, most commercially available adjustable ceiling monitor mounts can only support LCD monitors. Thus the ability to use an LCD monitor in a VEP recording system would be of benefit. However, as we have 